TITLE:

\title{
A game options approach to the investment problem with convertible debt financing
}

$\operatorname{AUTHOR}(S)$ :

Egami, Masahiko

CITATION:

Egami, Masahiko. A game options approach to the investment problem with convertible debt financing. Journal of Economic Dynamics and Control 2010, 34(8): 1456-1470

ISSUE DATE:

2010-08

URL:

http://hdl.handle.net/2433/126626

RIGHT:

(c) 2010 Elsevier B.V.; この論文は出版社版でありません。引用の際には 出版社版をご確認ご利用ください。; This is not the published version. Please cite only the published version. 


\title{
A Game Options Approach to the Investment Problem with Convertible Debt Financing
}

\author{
Masahiko Egami \\ Graduate School of Economics, Kyoto University ${ }^{1}$
}

\begin{abstract}
We consider a firm that operates a single plant and has an expansion option to invest in a new plant with convertible debt financing. This conversion feature introduces another complication not only because of the added conversion timing problem (by the bond holder) but also because the equity holder needs to take future conversion into account when evaluating her expansion/financing decision. We have two main objectives here. We use game options techniques to analyze optimal strategies involved in this convertible debt financed expansion problem. The first goal is to provide a comprehensive framework and procedure for solving the problem in a mathematically tractable way. Secondly, we illustrate our solution method through a concrete example with economic analysis. This includes a comparison with straight bond financing and comparative statics with respect to price volatility and conversion ratio. In this regard, we attempt to clarify how the conversion feature affects the equity holder's investment decisions. Throughout the paper, we study expansion options by viewing a firm's existing operation, bankruptcy threat, conversion decisions and financing decisions all together.
\end{abstract}

Key words: Convertible bond, Investment decision, Optimal stopping, Game options, JEL Classification: D81, D92, G31, G33.

Mathematics Subject Classification (2000) : Primary 60G40, 91A15: Secondary: 91B28.

\section{Introduction}

Convertible debt has a special feature such that the holder can convert to the issuing firm's equity. In this paper we focus on the role of convertible bonds when used to finance expansion/investment options of the firm. We provide a comprehensive framework in which one can find optimal strategies both for convertible bond holder and for equity holder. Among others, one can compute equity value equipped with the expansion option, convertible bond value with its coupon rate, and threshold values of conversion, expansion, and bankruptcy. Next, we compare equity value in convertible bond financing with that in straight bond. Moreover, by varying conversion ratios to zero, we discuss how coupon rates and expansion threshold levels (in the case of convertible bond financing) move toward those in the case of straight bond financing.

\subsection{Background}

In case of straight debt financing for expansion, several papers explore the interplay of financing and investment/operating policies in a dynamic decision environment and arrive at a different conclusion. Mello and Parsons [15] build on the contingent claims model of Brennan and Schwartz [2] of a mine to investigate how debt financing influences operating decisions and find that agency costs are comparable or greater than underwriting and other direct administrative expenses of leverage. Using a flexible discrete time, discrete state, finite horizon model, Childs et al. [3] show that the agency costs of asset substitution and underinvestment have a substantial effect on optimal leverage and firm value. In the construction of Childs et al. [3], the firm optimizes its initial leverage ratio. To study the overinvestment problem (that is, the firm invests earlier with debt financing than in the case

\footnotetext{
${ }^{1}$ Yoshida-honmachi, Sakyo-ku, Kyoto 606-8501, Japan. Tel:+81-75-753-3430, Fax: +81-75-753-3490, Email:egami@econ.kyoto-u.ac.jp. Preprint submitted to Elsevier

March 19, 2010
} 
of equity financing), Mauer and Sarkar [14] consider a firm whose only asset is a real option in the form of an opportunity to invest in a production facility. They optimize leverage, debt maturity, and timing of exercise of the option to find that the agency cost of debt is a significant fraction of firm value. It should be noted that Hennessy [9] empirically finds a statistically significant relationship between debt overhang and over-and underinvestment. Egami [6] considers the investment timing, the value of loan commitment, and agency cost associated under the assumption of straight bond financing in a unified fashion.

We mention few papers regarding convertible bond issues. Hennessy and Tserlukevich [10] analyze callable and convertible bond pricing via stochastic games. See also Sîrbu and Shreve [21]. The empirical research in Korkeamaki and Moore [12] shows the firms with high marginal benefit of waiting, that is, high cash flow volatility and high growth prospects tend to defer investment. Sarkar [19] examines the convertible call decision under corporate taxation and possible default prior to maturity. It is also interesting that Pong et al. [8] use the concept of multi-stage compound options to the valuation of convertible bonds. For convertible bond financing applied in the expansion/financing problem, only a few papers are available to the best of our knowledge. Lyandres and Zhdanov [13] study the expansion problem with equity financing for the firm that has convertible bond (and straight bond) in their capital structure. Yagi et al. [22] examine the investment timing problem with convertible debt where the firm has an expansion option as its sole asset and considers exercising it with convertible bond financing. Our paper here, asides from the new solution method employed, differs in the following: We find the coupon rates endogenously, which in turn makes a direct comparison (of equity value, threshold values) between convertible and straight bond financing possible. Moreover, we clarify how convertible bond behaves as if it were a straight bond when conversion ratios are small.

\subsection{Contributions to the literature}

Our first contribution to the corporate finance literature is to solve the expansion problem financed by a convertible bond in a rigorous manner for a general linear diffusion model. In our problem setup, both parties (equity and bond holders) can exercise their options. The equity holder has to take the convertible bond holder's future behavior into account in making investment/financing decisions. We manage to solve a game options valuation embedded in an optimal stopping problem (Proposition 2.2). Accordingly, mathematics involved here becomes more complex than the one in the straight bond financing problem. In doing so, we start with a general diffusion that describes the underlying price process and solve the problem in quite a general way. We directly find, along with the two parties' optimal strategies, the equity value and the convertible debt value. Our solution should be contrasted with the method relying on the smooth-fit principle: In the real options literature, many authors, including the ones as cited above, usually formulate necessary conditions that the value function is supposed to satisfy. This leads to a set of (sometimes quite a few) non-linear equations, which is often solved solely on some numerical procedures. The smooth-fit condition at the boundaries are imposed to determine the coefficients and boundary values involved in the set of equations. However, we have to keep in mind that the existence and uniqueness of the solution to the system of equations have to be proved. Furthermore, since there is no guarantee that the value function satisfies the smooth-fit principle at the boundaries, one has to verify the optimality of the constructed solution by proving the variational inequalities, which is again not a trivial task. See, for example, Seierstad [20] (Section 4.5), Øksendal [16] (Theorem 10.4.1) and Øksendal and Sulem [17]. In this respect, it should become extremely difficult to solve the current problem (with convertible debt financing) via the conventional method because one has to find at least four threshold values (bankruptcy, expansion, conversion and closure) and the number of the equations involved is supposed to increase accordingly. Hence it is not so clear whether the numerical solutions (including solvability) are really general or highly depend on the parameters. We emphasize that our approach is, therefore, free from these concerns.

With the general solution method in hand, as our second contribution, we analyze an example (using a geometric Brownian motion) to obtain a complete solution to the expansion-conversion problem. In the due course, we examine the underlying economic implications via some comparative statics:

- We find initial conditions, in terms of model parameters, for convertible debt financing to work and clarify its economic meanings. See Lemma 3.3 and the remarks that follow.

- When the expansion is funded by convertible bonds, the expansion threshold is lower than in the case of 
straight bond financing under almost all parameter sets we used ${ }^{2}$. A possible explanation to this phenomenon is that the hybrid feature leads to a reduction of coupon payment, which in turn expedites the expansion behavior of the equity holder (= borrower).

- We show a range of conversion ratios from 0.5 to 0 and analyze how a convertible bond "converges" to a straight bond in terms of coupon rates and expansion threshold values. For a fixed price volatility $\sigma$, as conversion ratio $\eta$ becomes smaller, coupon rates basically increases to the coupon rate of straight bond, reflecting the decline in the value of conversion option. At the same time, the conversion threshold becomes larger since the bond holder is better off by enjoying a higher coupon. As $\eta$ decreases, the expansion threshold also increases to the expansion threshold level of straight bond again due to the increasing coupon rate.

- Next we vary volatility levels of the underlying price process. Let us denote coupon rates as a function of volatility: $c(\sigma)$. For relatively high conversion ratios $(\eta=0.3 \sim 0.5)$, the coupon rate $c(\sigma)$ of convertible bond is decreasing in $\sigma$. This is primely because the increased volatility makes the conversion option more valuable and accordingly the bond holder is paid a lower coupon. In the case of straight bond financing, a higher risk level leads to a higher coupon rate (i.e., $c(\sigma)$ is increasing in $\sigma$ ). This point highly contrasts with the convertible bond. Since there is no conversion feature in this case, the increased risk of bankruptcy should be compensated by a larger coupon rate. A more interesting observation is in the range of smaller $\eta$ 's (i.e., $\eta=0 \sim 0.2$ ). We observe that in this range, the transition from the phenomenon of convertible bond (i.e., $c^{\prime}(\sigma)<0$ ) to that of straight bond (i.e., $c^{\prime}(\sigma)>0$ ) is happening.

- We compare the equity value in convertible bond financing with that in straight bond financing. The straight bond financing case has larger equity values than convertible bond financing due to the higher coupon rate which creates a larger tax shield. As we point out in the preceding paragraph, as the conversion ratio $\eta$ goes down, equity value becomes larger and closer to that in the case of straight bond financing. From this point of view, it is advantageous to issue straight bonds for expansion in the given parameter set. It follows, however, that equity holder may have to face a trade-off: If she offers a higher conversion ratio to a potential lender, this would be an easier sell, but her equity value (with the commitment to obtaining financing from that lender) should drop. This is especially the case if potential lenders have higher preference for the upside potential, namely, future shares in the expanded firm.

The remainder of the paper is organized as follows. In Section 2, we formulate an investment-conversion problem in a general setting and provide a procedure to derive an optimal solution. We maintain mathematical tractability so that we can obtain solutions as explicit as possible. To incorporate the conversion option included in the bond, we employ the idea of game options which are of American type with the feature that both of the option writer and holder can exercise each party's option at any time. See Kifer [11] as one of the first examples in finance. For example, if the holder exercises its option earlier than the writer (=seller), the payoff function to the holder is $(K-x)^{+}$where $K$ is the strike price and $x$ is the stock price. On the other hand, if the writer exercises earlier, the payoff function to the holder is $(K-x)^{+}+\varepsilon$ for some $\varepsilon>0$. We use the characterization of the value functions in optimal stopping and game options on the real line developed by Dynkin [5], Dayanik and Karatzas [4] and Ekström [7]. We illustrate our solution in a concrete example in Section 3.1 where we give detailed analysis by following the solution recipe in Section 2. After that, we discuss, in Section 3.2, extensive economic implications: We compare with straight bond financing to emphasize the hybrid feature of convertible bond, which is followed by some comparative statics with respect to the model parameters. In Section 3.3, we discuss possible future research topics along this line. Some technical points are collected in the appendix.

\section{Expansion funded by convertible securities}

In this section, we study the expansion problem with convertible securities financing in a rather general setting.

\footnotetext{
${ }^{2}$ For the exceptional case, see Section 3.1.D.
} 


\subsection{Model}

Let $(\Omega, \mathcal{F}, \mathbb{P})$ be a complete probability space with a standard Brownian motion $W=\left\{W_{t}: t \geq 0\right\}$. Let $\mathbb{F}=\left(\mathcal{F}_{t}\right)_{t \geq 0}$ be the natural filtration of $W$ and $\mathcal{S}$ be the set of $\mathcal{F}_{t}$-stopping times. Let us consider the diffusion process $X=\left\{X_{t}: t \geq 0\right\}$ in the following form:

$$
\mathrm{d} X_{t}=\mu\left(X_{t}\right) \mathrm{d} t+\sigma\left(X_{t}\right) \mathrm{d} W_{t}, \quad X_{0}=x,
$$

with state space $\mathcal{I} \subseteq \mathbb{R}$ and assume that the strong solution exists. The diffusion process $X$ above represents price process of the firm's output. We consider a pure equity firm generating cash flows at the rate of $x-\xi$. Currently, the value of the pure equity firm is equal to

$$
V_{1}(x)=\sup _{C \in \mathcal{S}} \mathbb{E}^{x}\left[\int_{0}^{C} e^{-\rho t}(1-\theta)\left(X_{t}-\xi\right) \mathrm{d} t+e^{-\rho C} \gamma\right]
$$

where $\theta \in[0,1]$ is a tax rate and $\rho \in \mathbb{R}_{+}$is a discount rate. We assume that the firm produces unit output. $C \in \mathcal{S}$ denotes the time of closing down the plant with the payoff of constant residual value $\gamma \in \mathbb{R}_{+}$. This firm considers expanding the facility into $a(>1)$ times, financing this expansion with a convertible bond. Let us denote the time of expansion-issuance by $\Gamma \in \mathcal{S}$. Let us assume that the holder of the convertible bond will receive the pre-determined fraction $\eta /(1+\eta), \eta \in \mathbb{R}_{+}$of the company equity upon conversion. That is, upon conversion, per a unit of share, a new share of fraction $\eta$ is issued and given to the bond holder. After the expansion funded by issuing the convertible bond, two things can happen: either the debt holder declares conversion or the equity holder (borrower) declares bankruptcy whichever occurs first. Let us denote the time of conversion and bankruptcy by $\Lambda$ and $B$, respectively. Under this setting, we have

$$
\Gamma \leq \Lambda \wedge B
$$

and on $\{B<\Lambda\}$,

$$
C=+\infty
$$

almost surely. That is, if the equity holder declares bankruptcy and turn the company over to the bond holder, she does not own the firm any more and shall not close the plant by her hand. For simplicity (and following the convention in the literature), let us suppose that the convertible debt is perpetual with principal $c / \rho$. Here $c$ is the contractual coupon rate per period of time. At this time $\Lambda$, the firm becomes all equity free from coupon payments and bankruptcy. Let us denote by $V_{3}(\cdot)$ the value of the pure equity firm after the expansion and conversion:

$$
V_{3}(x):=\sup _{\tau_{3} \in \mathcal{S}} \mathbb{E}^{x}\left[\int_{0}^{\tau_{3}} e^{-\rho t} a(1-\theta)\left(X_{t}-\xi\right) \mathrm{d} t+e^{-\rho \tau_{3}} b \gamma\right]
$$

where $b$ can be equal to $a$. That is, at time $\tau_{3}$ the expanded firm with all equity closes down the plant and receives the scrap value $b \gamma$. At time $B$, the equity holder, before conversion, may declare bankruptcy to avoid coupon payments and turn the firm over to the convertible bond holder. Note that at time $B$, the convertible bond is written off and the bond holder takes over the firm with the value $V_{3}\left(X_{B}\right)$ since the firm had already expanded before. After the issuance of the convertible bond, two things can happen, conversion or bankruptcy. When the bond holder evaluates the convertible bond, he may take the worst case scenario into account. Namely, it is natural for him to assume that the equity holder should choose the bankruptcy threshold so that the payoff from the equity holder to the bondholder shall be minimized. Then, the value of the convertible bond at time $t$ and $X_{t}=x$ can be defined by

$$
\begin{aligned}
D_{c}(t, x):=\sup _{\Lambda} \inf _{B} \mathbb{E}_{t, x} & {\left[1_{\{\Lambda<B\}}\left(\int_{t}^{\Lambda} c e^{-\rho(s-t)} \mathrm{d} s+e^{-\rho(\Lambda-t)} \frac{\eta}{1+\eta} V_{3}\left(X_{\Lambda}\right)\right)\right.} \\
+ & \left.1_{\{\Lambda>B\}}\left(\int_{t}^{B} c e^{-\rho(s-t)} \mathrm{d} s+e^{-\rho(B-t)}\left(V_{3}\left(X_{B}\right)-P\right)\right)\right]
\end{aligned}
$$


where $P$ is a constant bankruptcy cost. Note that we often write $D_{c}(x):=D_{c}(0, x)$ for brevity and that we simplify the notation by writing $\mathbb{E}_{t, x}[\ldots .]:.=\mathbb{E}\left[\ldots \mid X_{t}=x\right]$. This equation means that the debt holder attempts to maximize his payoff at conversion time $\Lambda$ while the equity holder chooses her bankruptcy time $B$ to minimize her payoff to the bond holder. While it is true that to minimize the payoff to bondholder is not equivalent to maximizing the payoff to the shareholder, for the moment, we assume that the equity holder uses this bankruptcy threshold level $B$ (see Remark 2.3 where we relax this assumption) and she solves

$V_{2}(x):=\sup _{\Gamma, C \in \mathcal{S}} \mathbb{E}^{x}\left[\int_{0}^{\Gamma \wedge C} e^{-\rho t}(1-\theta)\left(X_{t}-\xi\right) \mathrm{d} t+1_{\{C<\Gamma\}} e^{-\rho C} \gamma\right.$

$$
\left.+1_{\{\Gamma<C\}}\left(\int_{\Gamma}^{\Lambda \wedge B} e^{-\rho t}\left(a(1-\theta)\left(X_{t}-\xi\right)-c(1-\theta)\right) \mathrm{d} t+e^{-\rho \Lambda} \frac{1}{1+\eta} V_{3}\left(X_{\Lambda}\right) 1_{\{\Lambda<B\}}\right)\right]
$$

to determine an optimal expansion level $\Gamma$ and closure level $C$. Here $c>0$ is coupon rate to be determined within the model with (2.7) below.

Let us suppose the cost of expansion to be $I$. Appropriate accounting of bankruptcy risk requires equating investment cost $I$ with debt value at the investment time.

$$
I=D_{c}\left(0, X_{\Gamma}\right)
$$

Since the value of the debt $D_{c}(t, x)$ is given by (2.5), this equation determines coupon rate $c$ endogenously together with (2.6).

We summarize our problem chronologically here: the equity holder chooses whether she closes down the plant (and receives $\gamma$ ) or expand the plant $a$ times by issuing a convertible bond. If the bond is issued, then the equity holder can declare bankruptcy, while the bond holder converts his bond into equity and the firm becomes all-equity firm with value $V_{3}$.

\subsection{Solution}

Let the infinitesimal generator of the diffusion $X$ be $\mathcal{A}$. The o.d.e. $(\mathcal{A}-\rho) v(x)=0$ has two fundamental solutions, $\psi(\cdot)$, increasing and $\varphi(\cdot)$, decreasing. Define

$$
F(x):=\psi(x) / \varphi(x)
$$

and call a real valued function $u F$-concave on $\mathcal{I}$ if, for every $x \in[l, r] \subseteq \mathcal{I}$,

$$
u(x) \geq u(l) \frac{F(r)-F(x)}{F(r)-F(l)}+u(r) \frac{F(x)-F(l)}{F(r)-F(l)} .
$$

See the Appendix for mathematical review of the optimal stopping theory that uses this $F$-concave function.

We solve this problem backwards: $V_{3} \Rightarrow D \Rightarrow V_{2}$. First, let us denote by $J_{3}(\cdot)$ the function on the righthand side of (2.4). That is, $J_{3}(x):=\mathbb{E}^{x}\left[\int_{0}^{\tau_{3}} e^{-\rho t} a(1-\theta)\left(X_{t}-\xi\right) \mathrm{d} t+e^{-\rho \tau_{3}} b \gamma\right]$. It is, by (A.2), simplified to $J_{3}(x)-a g_{1}(x)=\mathbb{E}^{x}\left[e^{-\rho \tau_{3}}\left(b \gamma-a g_{1}\left(X_{\tau_{3}}\right)\right)\right]$ where

$$
g_{1}(x):=\mathbb{E}^{x}\left[\int_{0}^{\infty} e^{-\rho t}(1-\theta)\left(X_{t}-\xi\right) \mathrm{d} t\right]
$$

In a typical problem, the solution is in the form:

$$
V_{3}(x)= \begin{cases}b \gamma, & x \in(0, \underline{x}] \\ A \varphi(x)+a g_{1}(x), & x \in[\underline{x}, \infty)\end{cases}
$$

where $A$ is a positive constant determined uniquely along with $\underline{x}>0$. 
Next, we analyze the value of convertible debt $D_{c}(t, x)$ in (2.5). Denote, by $U(t, x)$, the right hand side of (2.5) without sup inf. After simplification using (A.2), we have

$$
\begin{aligned}
U(t, x):=\mathbb{E}_{t, x}[ & 1_{\{\Lambda<B\}}\left(\frac{c\left(1-e^{-\rho(\Lambda-t)}\right)}{\rho}+\frac{\eta}{1+\eta} e^{-\rho(\Lambda-t)} V_{3}\left(X_{\Lambda}\right)\right) \\
& \left.+1_{\{B<\Lambda\}}\left(\frac{c\left(1-e^{-\rho(B-t)}\right)}{\rho}+e^{-\rho(B-t)}\left(V_{3}\left(X_{B}\right)-P\right)\right)\right] .
\end{aligned}
$$

We set

$$
f_{1}(x):=\frac{\eta}{1+\eta} V_{3}(x)-\frac{c}{\rho} \quad \text { and } \quad f_{2}(x):=V_{3}(x)-P-\frac{c}{\rho} .
$$

For this problem, we apply the following characterization of the value function of game options:

Theorem 2.1 (Ekström [7] Theorem 2.4). Assume that both boundary points of the state space of $X$ are natural. Then the value function $D_{c}$ equals the smallest continuous function $v: \mathcal{I} \mapsto \mathbb{R}$ such that $f_{1} \leq v \leq f_{2}$ and $v / \varphi$ is $F$-concave in all intervals $J \in \mathcal{I}$ where $v<f_{2}$.

This theorem together with the proof of Theorem 2.2 in Ekström [7] and the general theory of optimal stopping of one dimensional diffusions implies in particular that optimal conversions time is characterized as hitting times of the diffusion. With the example in Section 3, we shall show a complete analysis for the convertible bond valuation.

Finally, let us denote by $J_{2}(\cdot)$ the function on the right-hand side of (2.6) and extensively use (A.2) with appropriate stopping times to obtain

$$
\begin{aligned}
J_{2}(x)=\mathbb{E}^{x}[ & g_{1}(x)-e^{-\rho(\Gamma \wedge C)} g_{1}\left(X_{\Gamma \wedge C}\right)+1_{\{C<\Gamma\}} e^{-\rho C} \gamma \\
& \left.+1_{\{\Gamma<C\}}\left(e^{-\rho \Gamma} g_{2}\left(X_{\Gamma}\right)-e^{-\rho(\Lambda \wedge B)} g_{2}\left(X_{\Lambda \wedge B}\right)+\frac{1}{1+\eta} e^{-\rho \Lambda} V_{3}\left(X_{\Lambda}\right) 1_{\{\Lambda<B\}}\right)\right],
\end{aligned}
$$

where

$g_{1}(x):=\mathbb{E}^{x}\left[\int_{0}^{\infty} e^{-\rho t}(1-\theta)\left(X_{t}-\xi\right) \mathrm{d} t\right] \quad$ and $\quad g_{2}(x):=\mathbb{E}^{x}\left[\int_{0}^{\infty} e^{-\rho t}\left(a(1-\theta)\left(X_{t}-\xi\right)-c(1-\theta)\right) \mathrm{d} t\right]$.

Set $\tau_{l}:=\inf \left\{t \geq 0 ; X_{t} \leq l\right\}$ and $\tau_{r}:=\inf \left\{t \geq 0 ; X_{t} \geq r\right\}$ and note that the following representation is available for any $l, r \in \mathcal{I}$ and $x \in[l, r]$ :

$$
\mathbb{E}^{x}\left[1_{\left\{\tau_{l}<\tau_{r}\right\}} e^{-\rho \tau_{l}}\right]=\frac{\psi(x) \varphi(r)-\psi(r) \varphi(x)}{\psi(l) \varphi(r)-\psi(r) \varphi(l)}, \quad \mathbb{E}^{x}\left[1_{\left\{\tau_{r}<\tau_{l}\right\}} e^{-\rho \tau_{r}}\right]=\frac{\psi(l) \varphi(x)-\psi(x) \varphi(l)}{\psi(l) \varphi(r)-\psi(r) \varphi(l)},
$$

where $\psi(\cdot)$ and $\varphi(\cdot)$ are defined above. We obtain these representations by solving $(\mathcal{A}-\rho) f(x)=0$ with boundary conditions $f(l)=1$ and $f(r)=0$ (resp., $f(l)=0$ and $f(r)=1$ ) for finding $\mathbb{E}^{x}\left[1_{\left\{\tau_{l}<\tau_{r}\right\}} e^{-\rho \tau_{l}}\right]$ (resp., $\left.\mathbb{E}^{x}\left[1_{\left\{\tau_{r}<\tau_{l}\right\}} e^{-\rho \tau_{r}}\right]\right)$. For later use, let us set

$$
h_{1}(x):=\mathbb{E}^{x}\left[1_{\{B<\Lambda\}} e^{-\rho B}\right] \quad \text { and } \quad h_{2}(x):=\mathbb{E}^{x}\left[1_{\{\Lambda<B\}} e^{-\rho \Lambda}\right] .
$$

In the analysis of convertible bond, we have found, among other things, the optimal threshold values of bankruptcy and conversion. Let us denote them by $B^{*}$ and $\Lambda^{*}$, respectively. Now we show how to compute the optimal expansion and closure levels:

Proposition 2.2. If state $\Gamma \in \operatorname{int}(\mathcal{I})$ is in $\left(B^{*}, \Lambda^{*}\right)$, then $V_{2}(x)$ in (2.6) is the solution to the following two-sided optimal stopping problem:

$$
V_{2}(x)=\sup _{C, \Gamma \in \mathcal{S}} \mathbb{E}^{x}\left[1_{\{C<\Gamma\}} e^{-\rho C}\left(\gamma-g_{1}\left(X_{C}\right)\right)+1_{\{\Gamma<C\}} e^{-\rho \Gamma} Q\left(X_{\Gamma}\right)\right]+g_{1}(x)
$$


where $B^{*}$ (bankruptcy threshold) and $\Lambda^{*}$ (conversion threshold) are to be found in the previous step for the analysis of $D_{c}(\cdot, \cdot)$ and

$$
Q(x):=g_{2}(x)-g_{1}(x)-g_{2}\left(B^{*}\right) h_{1}(x)+\left(\frac{1}{1+\eta} V_{3}\left(\Lambda^{*}\right)-g_{2}\left(\Lambda^{*}\right)\right) h_{2}(x) .
$$

Proof. See the Appendix for a proof.

Solution procedure: In summary, we solve the problem in the following steps:

A. Find $\psi, \varphi, F$ and $F^{-1}$ from the diffusion at hand.

B. Compute $g_{1}, g_{2}$ and $V_{3}$ functions, which give us $f_{1}$ and $f_{2}$ to be evaluated.

C. By Theorem 1, solve the game options problem for $D_{c}$ to find $B$ (bankruptcy) and $\Lambda$ (conversion), which in turn provide us with $h_{1}$ and $h_{2}$.

D. Solve the two-sided optimal stopping problem for $V_{2}$ by Proposition 2. This gives us $C$ (closing-out) and $\Gamma$ (expansion + debt issuance).

Note that we do not specify any diffusions as underlying state variable here.

It is worth comparing the results above to the case of straight-debt financing. After the expansion, the firm continues its operation until bankruptcy, the timing of which is chosen by the equity holder. Let us denote the coupon rate of the straight bond by $\bar{c}$. She solves

$$
S_{2}(x):=\sup _{\bar{B} \in \mathcal{S}} \mathbb{E}^{x}\left[\int_{0}^{\bar{B}} e^{-\rho t}\left(a(1-\theta)\left(X_{t}-\xi\right)-\bar{c}(1-\theta)\right) \mathrm{d} t\right],
$$

of which solution, in general, is given by

$$
S_{2}(x)=g_{2, \bar{c}}(x)-g_{2, \bar{c}}\left(\bar{B}^{*}\right) \frac{\varphi(x)}{\varphi\left(\bar{B}^{*}\right)}
$$

on $x \in\left(\bar{B}^{*}, \infty\right)$ and zero elsewhere. Note that this $\bar{B}^{*}$ is the optimal bankruptcy threshold value (after expansion) and $g_{2, \bar{c}}$ is the same $g_{2}$ function defined in (2.13) but to emphasize the fact we use distinct coupon rate $\bar{c}$, the additional subscript is attached. The debt value in our context is given by

$$
S B_{\bar{c}}(x)=\mathbb{E}^{x}\left[\int_{0}^{\bar{B}} \bar{c} e^{-\rho t} \mathrm{~d} t+e^{-\rho \bar{B}}\left(V_{3}\left(X_{\bar{B}}\right)-P\right)\right]=\left(V_{3}\left(\bar{B}^{*}\right)-P-\frac{\bar{c}}{\rho}\right) \mathbb{E}^{x}\left[e^{-\rho \tau_{\bar{B}}}\right]+\frac{\bar{c}}{\rho} .
$$

Now by letting $r \rightarrow k_{2}$ (i.e., to the right natural boundary of the state space) in (2.14) with the bounded convergence theorem and the fact that $\varphi\left(k_{2}-\right)=0$, we have $\lim _{r \uparrow k_{2}} \mathbb{E}^{x}\left[1_{\left\{\tau_{l}<\tau_{r}\right\}} e^{-\rho \tau_{l}}\right]=\frac{\varphi(x)}{\varphi(l)}$. Hence for $x \in\left(\bar{B}^{*}, \infty\right)$, we have

$$
S B_{\bar{c}}(x)=\left(V_{3}\left(\bar{B}^{*}\right)-P-\frac{\bar{c}}{\rho}\right) \frac{\varphi(x)}{\varphi\left(\bar{B}^{*}\right)}+\frac{\bar{c}}{\rho}
$$

and for $x \in\left(0, \bar{B}^{*}\right), S B_{\bar{c}}(x)=V_{3}\left(\bar{B}^{*}\right)-P$. As in the convertible bond problem, the coupon rate $\bar{c}$ shall be determined endogenously from

$$
I=S B_{\bar{c}}\left(X_{\bar{\Gamma}}\right)
$$

where $\bar{\Gamma}$ is given by solving

$$
\begin{aligned}
S_{1}(x): & =\sup _{\bar{C}, \bar{\Gamma}} \mathbb{E}^{x}\left[\int_{0}^{\bar{C} \wedge \bar{\Gamma}} e^{-\rho t}(1-\theta)\left(X_{t}-\xi\right) \mathrm{d} t+1_{\{\bar{C}<\bar{\Gamma}\}} e^{-\rho \bar{C}} \gamma+1_{\{\bar{\Gamma}<\bar{C}\}} e^{-\rho \bar{\Gamma}} S_{2}\left(X_{\bar{\Gamma}}\right)\right] \\
& =\sup _{\bar{C}, \bar{\Gamma}} \mathbb{E}^{x}\left[1_{\{\bar{C}<\bar{\Gamma}\}} e^{-\rho \bar{C}}\left(\gamma-g_{1}\left(X_{\bar{C}}\right)\right)+1_{\{\bar{\Gamma}<\bar{C}\}} e^{-\rho \bar{\Gamma}}\left(S_{2}\left(X_{\bar{\Gamma}}\right)-g_{1}\left(X_{\bar{\Gamma}}\right)\right)\right]+g_{1}(x)
\end{aligned}
$$

along with $\bar{C}$. 
Remark 2.3. Suppose that we do not use the bankruptcy level $B$ obtained from Theorem 2.1 but instead we want to solve

$$
\begin{aligned}
V_{2}(x)=\sup _{\Gamma, C, B \in \mathcal{S}} \mathbb{E}^{x} & {\left[\int_{0}^{\Gamma \wedge C} e^{-\rho t}(1-\theta)\left(X_{t}-\xi\right) \mathrm{d} t+1_{\{C<\Gamma\}} e^{-\rho C} \gamma\right.} \\
& \left.+1_{\{\Gamma<C\}}\left(\int_{\Gamma}^{\Lambda \wedge B} e^{-\rho t}\left(a(1-\theta)\left(X_{t}-\xi\right)-c(1-\theta)\right) \mathrm{d} t+e^{-\rho \Lambda} \frac{1}{1+\eta} V_{3}\left(X_{\Lambda}\right) 1_{\{\Lambda<B\}}\right)\right]
\end{aligned}
$$

to find $B$ as a maximizer of equity value. By using Proposition 2.2, it is to solve the problem parameterized by $B$ :

$$
V_{2}(x, B)=\sup _{C, \Gamma \in \mathcal{S}} \mathbb{E}^{x}\left[1_{\{C<\Gamma\}} e^{-\rho C}\left(\gamma-g_{1}\left(X_{C}\right)\right)+1_{\{\Gamma<C\}} e^{-\rho \Gamma} Q\left(X_{\Gamma}, B\right)\right]+g_{1}(x)
$$

where

$$
Q(x, B):=g_{2}(x)-g_{1}(x)-g_{2}(B) h_{1}(x, B)+\left(\frac{1}{1+\eta} V_{3}\left(\Lambda^{*}\right)-g_{2}\left(\Lambda^{*}\right)\right) h_{2}(x, B) .
$$

So, first we fix $B$ and find $V_{2}(x, B)$ for that $B$ and then maximize $V_{2}(x, B)$ over $B$ (possibly numerically).

Remark 2.4. It is interesting to compare the values $\Gamma$ and $\bar{\Gamma}$. Now, let us compare (2.16) and (2.21) since these equations are used to find $\Gamma$ and $\bar{\Gamma}$, respectively. The first terms of these equations are exactly the same. As to the second terms, we need to check (2.17) and (2.18). If $\Gamma \in(B, \Lambda)$,

$Q(x)-\left(S_{2}(x)-g_{1}(x)\right)=\left(\frac{1}{1+\eta} V_{3}\left(X_{\Lambda}\right)-g_{2}\left(X_{\Lambda}\right)\right) h_{2}(x)+\left(g_{2, \bar{c}}(\bar{B}) \frac{\varphi(x)}{\varphi(\bar{B})}-g_{2}\left(X_{B}\right) h_{1}(x)\right)+g_{2}(x)-g_{2, \bar{c}}(x)$,

the sign of which may not be easily detected. Hence the comparison of $\Gamma$ and $\bar{\Gamma}$ should be ambiguous.

Remark 2.5. Proposition 2.2 is a necessary condition. But our solution covers the cases when $X_{\Gamma} \in(B, \Lambda)$ is not true. For example, if we have $X_{\Gamma} \in(\Lambda, \infty)$, then at expansion the bond holder converts at the same time. In this case, with $h_{1}(x)=0$ and $h_{2}(x)=1$, we evaluate $Q\left(X_{\Gamma}\right)$ in (2.17) with $X_{\Gamma}=X_{\Lambda}$. It reduces to

$$
V_{2}(x)=\sup _{C, \Gamma} \mathbb{E}^{x}\left[1_{\{C<\Gamma\}} e^{-r C}\left(\gamma-g_{1}\left(X_{C}\right)\right)+1_{\{\Gamma<C\}} e^{-r \Gamma}\left(\frac{1}{1+\eta} V_{3}\left(X_{\Gamma}\right)-g_{1}\left(X_{\Gamma}\right)\right)\right]+g_{1}(x) .
$$

This makes sense because in this case $X_{\Gamma}$ is in the region of conversion and hence the bond holder immediately converts into equity. The equity holder ends up with the share of $\frac{1}{1+\eta}$ of the expanded firm value $V_{3}$. On the other hand, if $X_{\Gamma}$ is in $(0, B)$, then $h_{1}(x)=1$ and $h_{2}(x)=0$ and we evaluate $Q\left(X_{\Gamma}\right)$ with $X_{\Gamma}=X_{B}$ to find

$$
V_{2}(x)=\sup _{\Gamma} \mathbb{E}^{x}\left[e^{-r \Gamma}\left(-g_{1}\left(X_{\Gamma}\right)\right)\right]+g_{1}(x)
$$

since $C=\infty$ (see (2.3)). This is just the same problem as the one for $V_{1}$ with $\gamma=0$.

\section{An illustration for complete solutions}

\subsection{Solution}

We illustrate the preceding argument with a concrete example where the state process is a geometric Brownian motion:

$$
\mathrm{d} X_{t}=\mu X_{t} \mathrm{~d} t+\sigma X_{t} \mathrm{~d} W_{t}, \quad X_{0}=x .
$$

One of the main objectives here is to show a complete analysis of this rather complex problems. This should contrasts with the other papers that rely on a set of several non-linear equations and on numerical computations for finding solutions. 
A. Data: For simplicity, we treat $a=b$. As usual, we assume that $\mu<\rho$. The infinitesimal generator $\mathcal{A}$ of $X$ in this case is $\mathcal{A} f(x) \triangleq\left(\sigma^{2} / 2\right) x^{2} f^{\prime \prime}(x)+\mu x f^{\prime}(x)$ acting on a smooth function $f(\cdot)$. The o.d.e. $(\mathcal{A}-\rho) f(x)=0$ for this diffusion provides the fundamental solutions $\psi(x)=x^{p_{1}}$ and $\varphi(x)=x^{-p_{2}}$ with $p_{1}>1$ and $-p_{2}<0$ (that is, $\left.p_{2}>0\right)$, which are the two roots of $p(p-1) \sigma^{2} / 2+p \mu-\rho=0$. Hence we have $F(x)=\psi(x) / \varphi(x)=x^{p_{1}+p_{2}}$ and $F^{-1}(y)=y^{\frac{1}{p_{1}+p_{2}}}$. Let us write

$$
a^{\prime}:=a(1-\theta) .
$$

For (2.13), we have

$$
g_{1}(x)=(1-\theta)\left(\frac{x}{\rho-\mu}-\frac{\xi}{\rho}\right) \quad \text { and } \quad g_{2}(x)=\frac{a^{\prime} x}{\rho-\mu}-\frac{a^{\prime} \xi+(1-\theta) c}{\rho}
$$

explicitly and $V_{3}(x)$ in (2.9) has an explicit form with

$$
A=a^{\prime} \underline{x}^{p_{2}}\left(\gamma-\frac{\underline{x}}{\rho-\mu}+\frac{\xi}{\rho}\right) \quad \text { and } \quad \underline{x}=\frac{p_{2}}{1+p_{2}} \frac{(\xi+\rho \gamma)(\rho-\mu)}{\rho},
$$

which yields

$$
A=a^{\prime} \underline{x}^{p_{2}} \frac{\rho \gamma+\xi}{\rho\left(1+p_{2}\right)}>0 .
$$

Hence the $V_{3}$ function for this problem becomes

$$
V_{3}(x)= \begin{cases}a \gamma, & (0, \underline{x}), \\ A x^{-p_{2}}+a^{\prime}\left(\frac{x}{\rho-\mu}-\frac{\xi}{\rho}\right), & {[\underline{x}, \infty) .}\end{cases}
$$

B. Analysis of convertible bond: In our example, $U(0, x)$ in (2.10) contain two functions $f_{1}$ and $f_{2}$ of the following form:

$$
f_{1}(x):= \begin{cases}\frac{\eta}{1+\eta} a^{\prime} \gamma-\frac{c}{\rho}, & x \in(0, \underline{x}), \\ \frac{\eta}{1+\eta}\left(A x^{-p_{2}}+\frac{a^{\prime} x}{\rho-\mu}-\frac{a^{\prime} \xi}{\rho}\right)-\frac{c}{\rho}, & x \in[\underline{x}, \infty)\end{cases}
$$

and

$$
f_{2}(x):= \begin{cases}a^{\prime} \gamma-\frac{c}{\rho}-P, & x \in(0, \underline{x}), \\ A x^{-p_{2}}+\frac{a^{\prime} x}{\rho-\mu}-\left(\frac{a^{\prime} \xi}{\rho}+\frac{c}{\rho}+P\right), & x \in[\underline{x}, \infty) .\end{cases}
$$

Namely, $f_{1}$ is the reward (to the bond holders) when the equity holder declares bankruptcy and $f_{2}$ is the reward when the bond holders declare conversion.

By denoting $y:=F(x)$, in our example, $H_{1}:=f_{1} / \varphi \circ F^{-1}$ and $H_{2}:=f_{2} / \varphi \circ F^{-1}$ are explicitly available:

$$
H_{1}(y):= \begin{cases}\left(\frac{\eta}{1+\eta} a^{\prime} \gamma-\frac{c}{\rho}\right) \cdot y^{\frac{p_{2}}{p_{1}+p_{2}}}, & y \in[0, F(\underline{x})), \\ \frac{\eta}{1+\eta}\left(A+\frac{a^{\prime}}{\rho-\mu} y^{\frac{1+p_{2}}{p_{1}+p_{2}}}-\left(\frac{a^{\prime} \xi}{\rho}+\frac{c(1+\eta)}{\eta \rho}\right) y^{\frac{p_{2}}{p_{1}+p_{2}}}\right), & y \in[F(\underline{x}), \infty) .\end{cases}
$$

and

$$
H_{2}(y):= \begin{cases}\left(a^{\prime} \gamma-\frac{c}{\rho}-P\right) \cdot y^{\frac{p_{2}}{p_{1}+p_{2}}}, & y \in[0, F(\underline{x})), \\ A+\frac{a^{\prime}}{\rho-\mu} y^{\frac{1+p_{2}}{p_{1}+p_{2}}}-\left(\frac{a^{\prime} \xi}{\rho}+\frac{c}{\rho}+P\right) y^{\frac{p_{2}}{p_{1}+p_{2}}}, & y \in[F(\underline{x}), \infty) .\end{cases}
$$

On the set $\{0<x \leq \underline{x}\}$, consider $H_{2}(y)-H_{1}(y)$. It is easy to see that

$$
H_{1}(y)<H_{2}(y) \quad \Leftrightarrow \quad a^{\prime} \gamma>(1+\eta) P .
$$


On the set $\{x>\underline{x}\}$, it is a little more complicated:

$$
H_{1}(y)<H_{2}(y) \Leftrightarrow A+\frac{a^{\prime}}{\rho-\mu} y^{\frac{1+p_{2}}{p_{1}+p_{2}}}>\left(\frac{a^{\prime} \xi}{\rho}+(1+\eta) P\right) y^{\frac{p_{2}}{p_{1}+p_{2}}} .
$$

To analyze further on this region, let us check the shape of the functions $H_{i}(y)$ with $i=1,2$.

\section{Observation:}

(i) First, for a large $y$, the dominating terms are the ones with $y^{\frac{1+p_{2}}{p_{1}+p_{2}}}$. Since $\frac{\eta}{1+\eta}<1$, we must have $H_{1}(y)<$ $H_{2}(y)$ for a large $y$ by comparing the second parts of (3.5) and (3.6).

(ii) By a direct differentiation of the second parts of $H_{i}(y)$, it can be checked that $H_{i}(y)$ are decreasing up to $\hat{y}_{i}=F\left(\hat{x}_{i}\right), i=1,2$ where

$$
\hat{x}_{1}:=\left(\frac{a^{\prime} \xi}{\rho}+\frac{c(1+\eta)}{\eta \rho}\right) \cdot M \quad \text { and } \quad \hat{x}_{2}:=\left(\frac{a^{\prime} \xi}{\rho}+\frac{c}{\rho}+P\right) \cdot M
$$

by setting $M=: \frac{p_{2}(\rho-\mu)}{a^{\prime}\left(1+p_{2}\right)}>0$. Note then $\underline{x}=\left(\frac{a^{\prime} \xi}{\rho}+a^{\prime} \gamma\right) \cdot M$ and that

$$
\hat{x}_{2}<\hat{x}_{1} \Leftrightarrow P-\frac{c}{\rho \eta}<0 \text {. }
$$

Both functions are increasing on $\left[\hat{y}_{i}, \infty\right)$.

(iii) As to the second derivative, both functions (second part) are convex up to a certain point, say $\bar{y}_{i}$, greater than $\hat{y}_{i}$ and become concave thereafter.

At this point, we should make some qualitative observations on the parameters here. In particular, we may and do assume the following to make our further analysis economically meaningful:

Assumption 3.1. The $H_{1}(y)$ and $H_{2}(y)$ functions are negative in the region $y \in(0, F(\underline{x}))$ :

$$
\text { (a) } \frac{\eta}{1+\eta} a^{\prime} \gamma<\frac{c}{\rho} \text { and }(b) \quad a^{\prime} \gamma-P<\frac{c}{\rho} \text {. }
$$

Both (a) and (b) are quite reasonable assumptions due to the following justifications: Since $c / \rho=\int_{0}^{\infty} c e^{-\rho t} \mathrm{~d} t$, the first assumption is that, at the conversion, the convertible debt holder's share of the scrap value is smaller than the the value of the financing cost. In other words, the bond holder cannot retrieve $c / \rho$ by obtaining the scrap value. It means that the incentive to convert the bond into equity (to obtain $\left(\frac{\eta}{1+\eta}\right) a^{\prime} \gamma$ ) must be smaller than the possible interest income $c / \rho$. Similarly, the second assumption is that, at the bankruptcy, the scrap value of the expanded firm minus the bankruptcy cost is smaller than the value of financing costs, which means the convertible bond is risky. Under these assumptions it follows from (3.1) and (3.9) that

$$
\underline{x}<\hat{x}_{1} \quad \text { and } \quad \underline{x}<\hat{x}_{2} .
$$

Assumption 3.2. $a^{\prime} \gamma>(1+\eta) P$, that is $H_{1}(y)<H_{2}(y)$ on $y \in(0, F(\underline{x}))$.

Note that Assumption 3.2 means that

$$
\frac{\eta}{1+\eta} a^{\prime} \gamma<a^{\prime} \gamma-P
$$

which in turn implies that the bankruptcy cost $P$ is small enough for the bond holder to participate into this financing transaction. That is, the value at bankruptcy that the bond holder takes over is still greater than his share in the scrap value at closure. Note that with this extra assumption, we have $\hat{x}_{2}<\hat{x}_{1}$. Indeed, we have from Assumption 3.2 and from Assumption 3.1-(a)

$$
(1+\eta) P<a^{\prime} \gamma<\frac{c(1+\eta)}{\rho \eta} .
$$


The extreme values in the inequalities give us $P<\frac{c}{\rho \eta}$, which is (3.10). Note that Assumption 3.1-(b) also implies the same. See also comments in Remark 3.4.

Now we show that $H_{2}$ function dominates $H_{1}$ :

Lemma 3.3. Under Assumptions 3.1 and 3.2, $H_{1}(y)<H_{2}(y)$ in the entire region $y \in \mathbb{R}_{+}$.

Proof. In view of Observation-(i), if the two functions were to intersect in the region $y \in(F(\underline{x}), \infty)$, then they have to do so at least twice. In particular, at some point, say $y^{\prime}>F(\underline{x})$, we must have $H_{1}^{\prime}\left(y^{\prime}\right)>H_{2}^{\prime}\left(y^{\prime}\right)$. But this implies, by setting $H_{1}^{\prime}\left(y^{\prime}\right)>H_{2}^{\prime}\left(y^{\prime}\right)$ with direct differentiations of (3.5) and (3.6), that

$$
x^{\prime}:=\left(y^{\prime}\right)^{\frac{1}{p_{1}+p_{2}}}<\left(\frac{a^{\prime} \xi}{\rho}+(1+\eta) P\right) \cdot M<\left(\frac{a^{\prime} \xi}{\rho}+a^{\prime} \gamma\right) \cdot M=\underline{x},
$$

a contradiction to $y^{\prime}>F(\underline{x})$. Note that the second inequality is due to Assumption 3.2. Hence we conclude that in our example, $H_{1}(y)<H_{2}(y)$ in the entire $y \in \mathbb{R}_{+}$.

Remark 3.4. While it seems a reasonable assumption, if Assumption 3.2 were not true, then we would have $\frac{a \gamma}{1+\eta}<P$. If this is the case, mathematically feasible patterns are either

$$
a^{\prime} \gamma<\frac{c(1+\eta)}{\rho \eta}<\frac{c}{\rho}+P<(1+\eta) P \quad \Rightarrow \quad \hat{x}_{1}<\hat{x}_{2},
$$

or

$$
a^{\prime} \gamma<(1+\eta) P<\frac{c}{\rho}+P<\frac{c(1+\eta)}{\rho \eta} \Rightarrow \hat{x}_{1}>\hat{x}_{2}
$$

Since we have now completely identified the shapes of the two functions $H_{1}$ and $H_{2}$ and their relationship, we shall find the solution to (2.5) by invoking Theorem 2.1, which states that the value function in the transformed space, $W$ is the smallest continuous function such that (a) $H_{1} \leq W \leq H_{2}$ and (b) $W$ is concave in the region $W<H_{2}$. Hence for this particular problem, let us draw a line segment that is tangent to $H_{2}$ (at a small value of $y$ ) and to $H_{1}$ (at a larger value of $y$ ) and denote the points tangent to $H_{1}$ and $H_{2}$ by $F(\Lambda)$ and $F(B)$, respectively. Note that the line is tangent to $H_{2}$ in the region where $H_{2}$ is convex and that it is tangent to $H_{1}$ in the region where $H_{1}$ is concave. Let us define the tangent line by $W_{0}(y):=\beta y+\delta$ with $\beta, \delta \in \mathbb{R}$ to be determined uniquely. Then the pursued function $W$ in the transformed space must be $H_{2}$ itself up to $F(B)$, the tangent line $W_{0}$ on $[F(B), F(\Lambda)]$, and finally $H_{1}$ (concave there) on $[F(\Lambda), \infty)$ since this is the smallest continuous function that satisfies both (a) and (b).

In summary, the $W$ function in the transformed space and $D_{c}(0, x)=\varphi(x) W(F(x))+\frac{c}{\rho}$ in the original space are

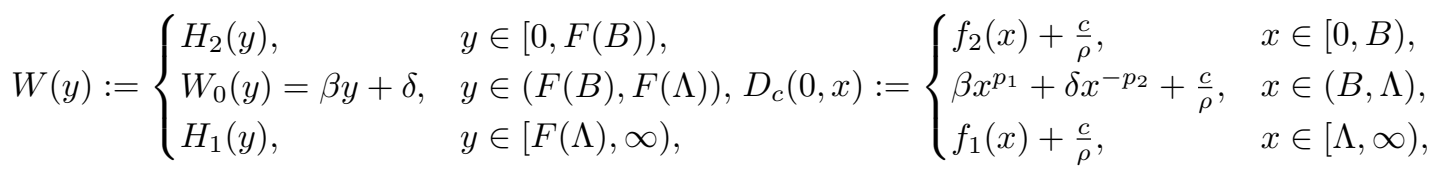

where $f_{1}, f_{2}, H_{1}$ and $H_{2}$ are defined in (3.3), (3.4), (3.5) and (3.6), respectively. While the unknowns are $\beta, \delta, B$ and $\Lambda$, we can take advantage of the facts that $\beta=H_{1}^{\prime}(F(\Lambda))=H_{2}^{\prime}(F(B))$ and effectively we have two equations for two unknowns: $H_{1}(F(\Lambda))-H_{2}(F(B))=H_{2}^{\prime}(F(B))(F(\Lambda)-F(B))$ and $H_{1}^{\prime}(F(\Lambda))=H_{2}^{\prime}(F(B))$. Then we necessarily have $\hat{x}_{2}<B$ and $\hat{x}_{1}<\Lambda$.

Before we conclude this step, we start a numerical example. The parameters are $a=b=2, \gamma=2, \xi=$ $0.15, \mu=0, \rho=0.045, \eta=0.4, \theta=0.3, I=35.071$ and $P=1.5$. With these data, we find $\underline{x}=0.106667$, $\hat{x}_{1}=1.7333$ and $\hat{x}_{2}=0.5643$ in the order of $\underline{x}<\hat{x}_{2}<\hat{x}_{1}$ as anticipated. Then we draw a tangent line both to $H_{1}$ and $H_{2}$ to obtain $W_{1}(y)=\beta y+\delta$ with $\beta=0.9245$ and $\delta=-13.4086$, which leads to $B=0.5805$ and 
$\Lambda=7.8146$.

C. Finding the optimal expansion strategy: Since we know optimal behavior of the convertible bond holder and bankruptcy policy of the equity holder associated with it, we are ready to solve for $V_{2}$ in (2.6). By using Proposition 2.2, let us call the reward function at bankruptcy and at expansion $f_{3}$ and $f_{4}$, respectively:

$f_{3}(x)=\gamma-(1-\theta)\left(\frac{x}{\rho-\mu}-\frac{\xi}{\rho}\right) \quad$ and $\quad f_{4}(x)=\frac{(1-\theta)(a-1) x}{\rho-\mu}-\frac{(1-\theta)(a-1) \xi+c(1-\theta)}{\rho}-m_{1} h_{1}(x)+m_{2} h_{2}(x)$

where $m_{1}:=g_{2}(B), m_{2}:=\frac{1}{1+\eta} V_{3}(\Lambda)-g_{2}(\Lambda)$ and $h_{1}, h_{2}$ are given by (2.15). Accordingly, we have the following reward functions in the transformed space:

$$
H_{3}(y):=(1-\theta)\left(\gamma+\frac{\xi}{\rho}\right) y^{\frac{p_{2}}{p_{1}+p_{2}}}-\frac{1-\theta}{\rho-\mu} y^{\frac{1+p_{2}}{p_{1}+p_{2}}}
$$

and

$H_{4}(y):=-\frac{(1-\theta)(a-1) \xi+c(1-\theta)}{\rho} y^{\frac{p_{2}}{p_{1}+p_{2}}}+\frac{(1-\theta)(a-1)}{\rho-\mu} y^{\frac{1+p_{2}}{p_{1}+p_{2}}}-\frac{m_{1}}{k}(\varphi(\Lambda) y-\psi(\Lambda))+\frac{m_{2}}{k}(\psi(B)-\varphi(B) y)$

where we used the representations in (2.14) with $k:=\psi(B) \varphi(\Lambda)-\psi(\Lambda) \varphi(B)<0$. By direct computations we observe

(a) $H_{3}(y)$ is first increasing and concave and then decreases and becomes convex.

(b) Since $\lim _{y \downarrow 0} H_{4}^{\prime}(y)<0$ and $\lim _{y \rightarrow \infty} H_{4}^{\prime}(y)>0, H_{4}(y)$ must change signs at least once. [We used $a>1$.] But the monotonicity of the derivatives of $y^{\frac{p_{2}}{p_{1}+p_{2}}}$ and $y^{\frac{1+p_{2}}{p_{1}+p_{2}}}$ implies that $H_{4}^{\prime}(y)$ could change signs at most once. Therefore, $H_{4}^{\prime}(y)$ changes signs exactly once. Moreover, the signs of $H_{4}^{\prime \prime}(y)$ tell us that $H_{4}(y)$ is first decreasing and convex and then increases and becomes concave.

(c) (a) and (b) imply that neither function dominates the other.

Therefore, the smallest concave majorant of $\max \left(H_{3}(y), H_{4}(y)\right)$ is $H_{3}(y)$ itself for small $y$, a line segment, call it $\hat{W}_{0}(y)$, tangent to $H_{3}$ and simultaneously to $H_{4}$, and finally $H_{4}(y)$ itself for large $y$. We provide $V_{2}(x)$ of this problem now:

$$
V_{2}(x)= \begin{cases}\gamma, & x \in[0, C], \\ \beta^{\prime} x^{p_{1}}+\delta^{\prime} x^{-p_{2}}+(1-\theta)\left(\frac{x}{\rho-\mu}-\frac{\xi}{\rho}\right), & x \in(C, \Gamma), \\ a^{\prime}\left(\frac{x}{r-\mu}-\frac{\xi}{\rho}\right)-\frac{(1-\theta) c}{\rho}-m_{1} \cdot h_{1}(x)+m_{2} \cdot h_{2}(x), & x \in[\Gamma, \infty),\end{cases}
$$

where $h_{1}(x)=\frac{\psi(B) \varphi(x)-\psi(x) \varphi(B)}{k}$ and $h_{2}(x)=\frac{\psi(x) \varphi(\Lambda)-\psi(\Lambda) \varphi(x)}{k}$ from (2.15). We can determine $\beta^{\prime}$ and $\delta^{\prime}$ uniquely as the slope and intercept of the line $\hat{W}_{0}$, respectively.

D. Economic Implications: We revisit the numerical example with the same parameter set. See Figure 1. The resulting thresholds are $C=0.1194<B$ and $\Gamma=3.1138<\Lambda$. Note that for the purpose of comparing with straight bond financing, we determine the bankruptcy threshold by following Remark 2.3 and so it is recomputed as $B=0.3795$. Along with them, we obtain $\beta^{\prime}=2.1780>\beta$ and $\delta^{\prime}=0.4435>\delta$. Hence we have $\Gamma \in(B, \Lambda)$, which provides us with the following scenario:

Suppose that the price level $x$ is in $(C, \Gamma)$.

1. If the diffusion hits $\Gamma$ earlier than $C$, then the equity holder issues a convertible bond to finance the proposed expansion. This point is in the continuation region of the conversion problem, so that the debt holder should not declare conversions upon the expansion and issuance of the convertible bond (at time $\Gamma$ ). Then there are two cases: 


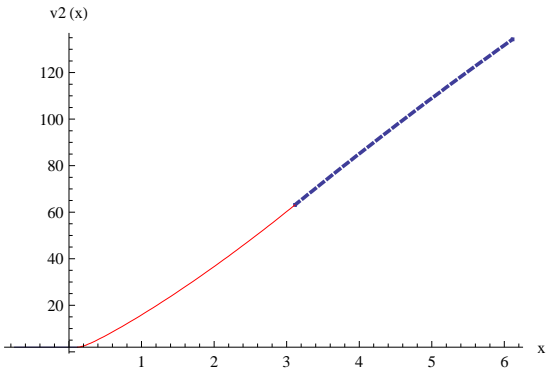

(a)

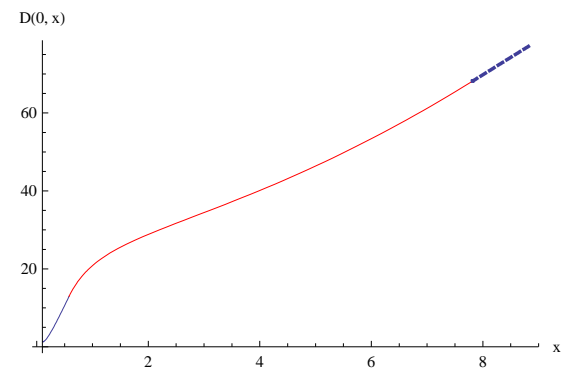

(b)

Figure 1: Equity value and convertible debt value (a) The value function $V_{2}(x)$ consists of three parts. (b) The value of the convertible bond $D_{c}(0, x)$.

Table 1: Summary of the optimal strategies: Expansion financed by convertible and straight bonds

\begin{tabular}{|c|c|c|c|}
\hline & Expansion & Conversion & Termination \\
\hline \hline Convertible Bond & {$\left[V_{2},(2.6)\right]$} & {$\left[D_{c},(2.5)\right]$} & {$\left[V_{3},(2.4)\right]$} \\
$c=1.5$ & $C=0.1194$ & $B=0.3795$ & $\underline{x}=0.1067$ \\
& $\Gamma=3.1138$ & $\Lambda=7.8146$ & \\
\hline Straight Bond & {$\left[S_{1},(2.21)\right]$} & N.a. & {$\left[S_{2},(2.18)\right]$} \\
$\bar{c}=1.845$ & $\bar{C}=0.1182$ & & $\bar{B}=0.4767$ \\
& $\bar{\Gamma}=3.7819$ & & \\
\hline
\end{tabular}

(i) If the diffusion hits $\Lambda=7.8146$ earlier than $B=0.3795$, then the bond holder declares conversion. The firm becomes an all-equity entity and continues until the diffusion hits $\underline{x}=0.10667$.

(ii) If the diffusion instead hits $B$ first, then the equity holder declares bankruptcy and turn the firm over to the bond holder. The bond holder pays $P$ out of his pocket and continues the operation until the diffusion hits $\underline{x}$. Note that we have $\underline{x}<B$ as shown above in step $\mathbf{B}$.

2. If the diffusion hits $C$ prior to $\Gamma$, then the equity holder closes down the plant and hence nothing happens.

Note that the coupon rate for the convertible bond is $c=1.5$ via (2.7). The argument up to this point provides a solution to the problem.

E. Comparison with straight-debt financing: Let us summarize the values obtained from this example: Recall that the parameters are $a=2, \gamma=2, \xi=0.15, \mu=0, \rho=0.045, \eta=0.4, \theta=0.3, I=35.071$ and $P=1.5$. Table 1 shows the threshold values. We also put function names and equation numbers in the brackets. Let us now compare our example with straight bond financing. In this particular case, we have

$$
\bar{B}=\frac{p_{2}}{1+p_{2}} \frac{\left(a^{\prime} \xi+\bar{c}(1-\theta)\right)(\rho-\mu)}{a^{\prime} \rho} .
$$

for $\bar{B}$ in (2.18). We compute the value of straight bond coupon rate $\bar{c}=1.845$ that satisfies (2.20) and is greater than $c=1.5$ as expected. We check the expansion threshold $\bar{\Gamma}$ in (2.21) to find $\bar{\Gamma}=3.7819>3.1138=\Gamma$ for this parameter set. The value of the straight bond $S B_{\bar{c}}(x)$ in (2.19) with this $\bar{c}$ is computed and compared with the value of the convertible bond $D_{c}(x)$ in Figure 2-(a). Figure 2-(b) displays the total firm value (debt plus equity) of the two cases. Note that the value of the equity at the expansion in the convertible bond is $V_{2}(x)$ in (2.16) evaluated at $\Gamma=0$, which yields $Q(x)+g_{1}(x)$. The straight bond financing gives us a greater firm value. This difference comes from the larger tax shield created by the greater coupon rate in the straight bond financing.

Figure 3 compares the equity value of convertible bond financing $V_{2}(x)$ with that of straight bond financing $S_{1}(x)$. The straight bond financing case has larger equity values than the convertible bond financing. The main 


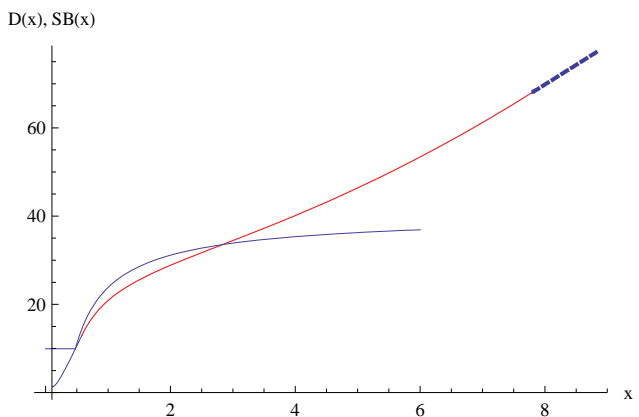

(a)

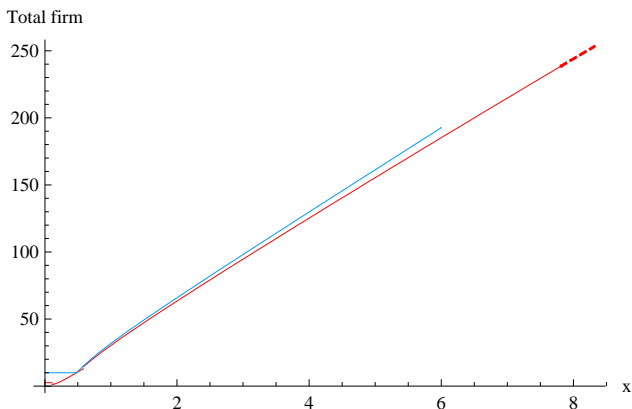

(b)

Figure 2: Comparison of $D_{c}$ (red) and $S B_{\bar{c}}$ (blue): (a) For small $x, S B_{\bar{c}}(x)$ is greater than $D_{c}(x)$. But for a larger $x, D$ curve dominates $S B$ curve due to the increasing possibility of conversion. (b) The total firm values (debt+equity) are compared between convertible and straight bond. The upper curve (blue) represents the straight bond plus the equity value at the expansion. The lower curve (lower) represents the convertible bond plus the equity value. We can check the distance between the two curves converge to zero as the tax rate goes to zero for $x$ greater than the bankruptcy levels (since the Modigliani-Miller theorem holds then).

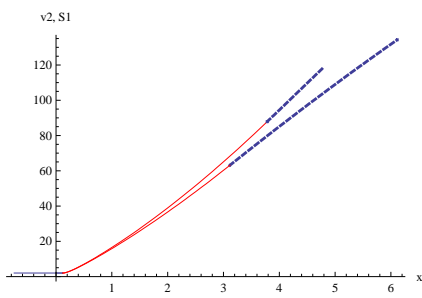

(a)

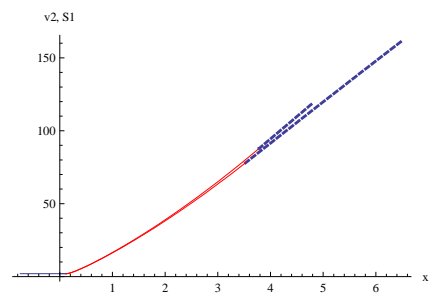

(b)

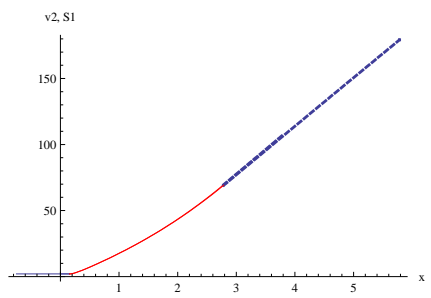

(c)

Figure 3: Equity values by straight bond and by convertible bond financing (a) The equity value $S_{1}(x)$ (straight debt financing) is above $V_{2}(x)$ (convertible debt financing) but when the option value is small (and the interest rate is higher), then the two values become closer. (a) $\theta=0.3, \eta=0.4$ and $\sigma=0.25$. (b) $\theta=0.3, \eta=0.2$ and $\sigma=0.25$. Figure (c) shows that the two values are almost identical with $\theta=0.15, \eta=0.125$ and $\sigma=0.15$.

reason seems due to the higher coupon rate in the latter case $(\bar{c}=1.845)$, which creates a larger tax shield (See Figure 3-(a)). However, when the conversion ratio $\eta$ goes down to 0.2 from 0.4 . The embedded conversion option value becomes smaller and this is compensated by a higher coupon rate (from $c=1.5$ to 1.712: see Table 2). As a consequence, the equity value with $\eta=0.2$ becomes larger and closer to that of straight bond financing. See Figure 3-(b). Since the tax shield seems to play an important role in this comparison, we reduce the tax rate from $\theta=0.3$ to $\theta=0.15$. We observe that the two curves, $V_{2}(x)$ and $S_{1}(x)$ become closer after this change. Moreover, Figure 3-(c) shows that the two equity values are almost identical with $\theta=0.15$ by using $\sigma=0.15$ and $\eta=0.125$. We have not encountered the case where the order is reversed. When the tax rate has decreased, both $c$ and $\bar{c}$ become smaller. While $c$ declines greater relative to $\bar{c}$, the relationship $c<\bar{c}$ is not reversed in our parameter sets. ${ }^{3}$ However, since the results rely on several factors, we cannot deny the possibility that the order would be reversed in a sufficiently low tax rate coupled with appropriate parameters of volatility, conversion ratio, etc.

Now take a look at Table 2 where we see the threshold values and coupon rates with varying $\eta$ and $\sigma$. We discuss comparative statics in the next subsection, but in this subsection, we focus on the issue of convertible versus straight bond. If we fix the parameter $\sigma$ and view horizontally, for each row, we see "convergence" of $\Gamma$ 's and coupon rates as $\eta$ becomes closer to zero. In relation to this, observe that $\Lambda$ becomes very large relative to $\Gamma$ as $\eta$ approaches zero. This is another evidence of convertible bond behaving as if it were straight bond. That is, the impact of conversion feature is very weak since it takes a lot of time (or perhaps unlikely) to reach that high price level. Moreover, the small ratio $\eta=0.1$ combined with $\sigma=0.15$, we observe that $c$ is slightly larger than $\bar{c}$ and

\footnotetext{
${ }^{3}$ In the case illustrated in Figure 3-(c), we have $c=1.652$ and $\bar{c}=1.664$.
} 
Table 2: Comparative statics

\begin{tabular}{|c||c||c|c|c|c|c|}
\hline & Straight Debt & $\eta=0.1$ & $\eta=0.2$ & $\eta=0.3$ & $\eta=0.4$ & $\eta=0.5$ \\
\hline$\sigma=0.15$ & $\bar{c}=1.677$ & $c=1.689$ & $c=1.667$ & $c=1.633$ & $c=1.589$ & $c=1.543$ \\
& $\Gamma=2.8172$ & $\Gamma=2.8387$ & $\Gamma=2.8040$ & $\Gamma=2.7503$ & $\Gamma=2.6808$ & $\Gamma=2.6083$ \\
& & $\Lambda=21.9154$ & $\Lambda=11.8266$ & $\Lambda=8.3727$ & $\Lambda=6.5793$ & $\Lambda=5.4729$ \\
& $c / \rho=37.27$ & $c / \rho=37.53$ & $c / \rho=37.04$ & $c / \rho=36.29$ & $c / \rho=35.31$ & $c / \rho=34.29$ \\
\hline$\sigma=0.25$ & $\bar{c}=1.845$ & $c=1.813$ & $c=1.712$ & $c=1.603$ & $c=1.500$ & $c=1.407$ \\
& $\Gamma=3.7819$ & $\Gamma=3.7192$ & $\Gamma=3.5233$ & $\Gamma=3.3125$ & $\Gamma=3.1138$ & $\Gamma=2.9350$ \\
& & $\Lambda=31.0652$ & $\Lambda=15.7019$ & $\Lambda=10.4639$ & $\Lambda=7.8146$ & $\Lambda=6.2202$ \\
& $c / \rho=41.00$ & $c / \rho=40.29$ & $c / \rho=38.04$ & $c / \rho=35.07$ & $c / \rho=33.33$ & $c / \rho=31.27$ \\
\hline$\sigma=0.35$ & $\bar{c}=2.060$ & $c=1.912$ & $c=1.698$ & $c=1.518$ & $c=1.370$ & $c=1.252$ \\
& $\Gamma=4.9831$ & $\Gamma=4.6543$ & $\Gamma=4.1576$ & $\Gamma=3.7395$ & $\Gamma=3.3971$ & $\Gamma=3.1248$ \\
& & $\Lambda=40.8733$ & $\Lambda=19.0171$ & $\Lambda=11.9370$ & $\Lambda=8.5146$ & $\Lambda=6.5535$ \\
& $c / \rho=45.78$ & $c / \rho=42.89$ & $c / \rho=37.73$ & $c / \rho=33.73$ & $c / \rho=30.44$ & $c / \rho=27.82$ \\
\hline
\end{tabular}

$\Gamma>\bar{\Gamma}$. We report that in this case the equity values $\left(V_{2}(x)\right.$ and $\left.S_{1}(x)\right)$ are almost identical. In the other cases, that is, in reasonably high price volatility levels and/or reasonable conversion ratios, we observe $S_{1}(x)>V_{2}(x)$ for $x \in \mathbb{R}_{+}$. Since the optimization takes care of all future cash flows in finding threshold values, as a consequence, the comparison between the two financing boils down to coupon rates derived in the processes ${ }^{4}$. From this point of view, it is advantageous to issue straight bond for expansion. It follows, however, the equity holder may face a trade-off: If she offers a higher conversion ratio to a potential lender, this would be an easier sell, but her equity value (with the commitment to obtaining financing from that lender) should drop. This is especially the case if potential lenders have higher preference for the upside potential, namely, future shares in the expanded firm. See Figure 1-(b) for a rapid increase in the bond value as price level becomes closer to the threshold level.

\subsection{Comparative statics}

Let us take a look at Table 2 again.

Price Risk: We vary the volatility coefficient $\sigma$, maintaining all the other parameters (including $I=35.071$ ) unchanged. Let us denote $c(\sigma)$ and $\bar{c}(\sigma)$ as functions of $\sigma$. Some interesting phenomena characteristic of convertible bond financing are apparent. For the range of $\eta=0.3 \sim 0.5$, the coupon rate $c(\sigma)$ is decreasing in $\sigma$. This is primely because the increased volatility makes conversion option more valuable and accordingly the bond holder is paid a lower coupon. In the case of straight bond financing, a higher risk level leads to a higher coupon rate. That is, $c(\sigma)$ is increasing in $\sigma$. This point highly contrasts with the convertible bond. Since there is no conversion feature in this case, the increased risk of bankruptcy should be compensated by the larger coupon rate. Hence $\bar{c}$ increases as the volatility increases.

A more interesting observation ${ }^{5}$ is in the range of $\eta=0.1$ and $\eta=0.2$. In the column of $\eta=0.2$, we have $c(0.15)<c(0.25)$ and $c(0.25)>c(0.35)$. Going further, in the column of $\eta=0.1$, we have $c(0.15)<$ $c(0.25)<c(0.35)$ which is the same order of $\bar{c}(0.15)<\bar{c}(0.25)<\bar{c}(0.35)$. Heuristically speaking, in this range of conversion ratios the "transition" from convertible to straight bond is happening. In this range, conversion option value is still there but is diminishing while the risk is increasing as $\sigma$ gets larger.

Both of the threshold values $\Gamma$ and $\Lambda$ increase with a higher volatility. This is consistent with the general result of optimal stopping problems. See, for example, Alvarez [1]. A brief explanation for this is that a high volatility of the diffusion leads to smaller amount of time required to reach any state. The effect of discounting shall then be

\footnotetext{
${ }^{4}$ Conversion threshold values are so determined to maximize the debt value. Then, the distribution between coupon revenue and conversion option values is determined. In turn, given the coupon rate and bond holder's strategy, expansion threshold is so determined to maximize equity value, which is significantly affected by the tax shield created by coupon payments.

${ }^{5}$ The author thanks Takashi Shibata for the discussion on this point.
} 
reduced and we can get higher rewards by going further within the same amount of time. Hence the continuation region expands. On the other hand, the decreased coupon level gives the equity holder more incentive to borrow and expand earlier. In this particular problem, this second effect of the higher volatility seems smaller than the first one (due to the speeded-up diffusion). The overall $\Gamma$ increases as the volatility gets larger.

Moreover, we observe $\Gamma<\bar{\Gamma}$ except in the case of $\eta=0.1$ and $\sigma=0.15$ as discussed above. Economically speaking, the lower coupon rate (relative to $\bar{c}$ ) gives an incentive for the equity holder to borrow (and thereby expand) earlier. In summary, the conversion feature lowers the coupon rate and leads to early investment. While the settings are quite different, our result here is basically the same as Lyandres and Zhdanov [13] in the sense that convertible securities have the effect of accelerating the investment timing.

We also compute the expansion thresholds in the case of equity financing as a benchmark. For the values of $\sigma=0.15,0.25$ and 0.35 , in the equity financing case, $\Gamma=3.9431,5.3968$ and 7.8657 , respectively. We can confirm that in the presence of tax shield the debt financing prompts the equity holder to invest early.

Conversion ratio: Let us view Table 2 horizontally this time. Keep $\sigma$ fixed at 0.25 . When $\eta$ gets smaller, as expected, the coupon rate $c$ increases reflecting the decline in the value of conversion option. At the same time, the conversion threshold $\Lambda$ increases. It can be inferred that the decline of the conversion option value delays the bond holder's conversion since he is better off by enjoying a higher coupon. Now let us turn to the effect of fraction $\eta$ on the expansion threshold $\Gamma$. It increases slightly but consistently. The increased coupon payments should work as a disincentive for the equity holder to borrow/invest early. Note that the effect on $\Lambda$ is much bigger than on $\Gamma$ as can be seen from $\Lambda-\Gamma$ increases rapidly as $\eta$ gets smaller. It is natural because the effect of conversion ratio has a stronger impact on the bond holder than on the equity holder.

Table 2 can be useful because the bond holder and equity holder can consult it and negotiate financing terms. For example, in accordance with the price risk level, they can negotiate over conversion fractions and obtain information for each party's optimal strategies. The bond value at issuance is equal to $I=35.071$. We compute the present value of perpetual coupon income $c / \rho$, which is larger than $I$ when $\sigma$ is low and/or $\eta$ is low. That is, when the value of conversion option is small and consequently the bankruptcy risk has to be compensated by a higher coupon. As the option value gets larger, $c / \rho$ gets smaller accordingly.

Bankruptcy cost: We checked the effect of bankruptcy cost and report the bankruptcy cost $P$, which naturally leads to a higher coupon rate. From the bond holder's point of view, the increased coupon level delays his conversion ( $\Lambda$ increases). From the equity holder's point of view, the incentive to borrow early is smaller ( $\Gamma$ increases). In the case of $P=1.99$, we have $c=1.505, \Lambda=7.8152$ and $\Gamma=3.1220$.

\subsection{Concluding remarks with possible extensions}

The general framework and procedure based on the game options approach in Section 2 can be used in other versions of the investment problem. First, the diffusion does not have to be a geometric Brownian motion. For example, the Ornstein and Uhlenbeck process could model output prices of durable goods with cyclical tendencies. While the procedure is the same as in Section 3, the analysis of the reward function in the transformed space can be more technical. Second, the callable (by the issuer) feature can be added to the analysis in a rigorous manner. Third, one can extend the technique developed here to an expansion problem where the firm has an outstanding convertible bond. If the expansion is financed by a new equity, a dilution issue will occur as in Lyandres and Zhdanov [13] to the existing convertible bond holder as long as the expansion (along with issuance of new equity) occurs prior to conversion. Note that however, the problem reduces to the analysis of the convertible bond in Section 2. It is because our bond holder in Section 2 comes into this transaction at the same time as the expansion. In this scenario, we can solve it while keeping it in mind that the dilution effect be taken into consideration.

Acknowledgement: This research is in part supported by Grant-in-Aid for Scientific Research (C) No. 20530340, Japan Society for the Promotion of Science. The author is grateful to the anonymous Associate Editor and referees for constructive comments that help to improve the manuscript and also to Takashi Shibata, Kyoko Yagi and Ryuta Takashima for valuable discussions. 


\section{A. Appendix}

\section{A.1. Mathematical Review}

We shall extensively use the following property: Let $\beta \geq 0$ be a real constant and $f(\cdot)$ be a continuous function that satisfies

$$
\mathbb{E}^{x}\left[\int_{0}^{\infty} e^{-\beta t}\left|f\left(X_{t}\right)\right| \mathrm{d} t\right]<\infty,
$$

under which, for any stopping time $\tau \in \mathcal{S}$, we have

$$
\mathbb{E}^{x}\left[\int_{0}^{\tau} e^{-\beta t} f\left(X_{t}\right) \mathrm{d} t\right]=g(x)-\mathbb{E}^{x}\left[e^{-\beta \tau} g\left(X_{\tau}\right)\right]
$$

with

$$
g(x):=\mathbb{E}^{x}\left[\int_{0}^{\infty} e^{-\beta t} f\left(X_{t}\right) \mathrm{d} t\right]
$$

by the strong Markov property of $X$. We briefly review the general theory of optimal stopping problem by Dynkin [5] and Dayanik and Karatzas [4].

Let the infinitesimal generator of the diffusion $X$ be $\mathcal{A}$. The o.d.e. $(\mathcal{A}-\beta) v(x)=0$ has two fundamental solutions, $\psi(\cdot)$, increasing and $\varphi(\cdot)$, decreasing. Note that $\varphi\left(k_{1}+\right)=\psi\left(k_{2}-\right)=\infty$ and $\psi\left(k_{1}+\right)=\varphi\left(k_{2}-\right)=0$ for $k_{1}<k_{2}$ natural boundaries of the diffusion. (The diffusion cannot reach natural boundaries: for example in the case of geometric Brownian motion, $k_{1}=0$ and $k_{2}=\infty$.) Define

$$
F(x):=\psi(x) / \varphi(x)
$$

and call a real valued function $u F$-concave on $\mathcal{I}$ if, for every $x \in[l, r] \subseteq \mathcal{I}$,

$$
u(x) \geq u(l) \frac{F(r)-F(x)}{F(r)-F(l)}+u(r) \frac{F(x)-F(l)}{F(r)-F(l)} .
$$

Consider the optimal stopping problem with reward function $h$ defined and continuous on some $[c, d] \in \mathcal{I}$ :

$$
V(x):=\sup _{\tau \in \mathcal{S}} \mathbb{E}^{x}\left[e^{-\rho \tau} h\left(X_{\tau}\right)\right] .
$$

The value function is the smallest nonnegative majorant of $h(\cdot)$ such that $V(\cdot) / \varphi(\cdot)$ is $F$-concave on $[c, d]$. That is,

1. If $W(\cdot)$ is the smallest nonnegative majorant of $H:=(h / \varphi) \circ F^{-1}$ on $[F(c), F(d)]$, then $V(x)=$ $\varphi(x) W(F(x))$.

2. Define

$$
S \triangleq\{x \in[c, d]: V(x)=h(x)\}, \quad \text { and } \quad \tau^{*} \triangleq \inf \left\{t \geq 0: X_{t} \in S\right\},
$$

then $\tau^{*}$ is an optimal stopping rule.

Hence the optimal strategy is characterized as hitting times of the diffusion to some state in $\mathcal{I}$. For this reason, if we find the optimal bankruptcy timing $B$ as equal to $B=\inf \left\{t \geq 0: X_{t}=B^{*}\right\}$ for some point $B^{*} \in \mathcal{I}$, then we write $B^{*}=X_{B^{*}}$ when there is no risk of confusion.

To show the existence of a finite value function $v$, we just need to show the existence of a finite concave majorant $W$ by examining $H$. Accordingly, the major task now reduces to analyzing the function $H$ in the transformed space. The following summarizes the procedure:

1. Define the increasing function $F(x)=\psi(x) / \varphi(x)$ and transform $h$ to $H=\frac{h}{\varphi} \circ F^{-1}$,

2. Identify the smallest concave majorant of $H$ and call it $W$,

3. Find the points where $H$ and $W$ meet to obtain the optimal boundaries in the transformed space, and

4. Transform back to recover the value function by $v(x)=\varphi(x) W(F(x))$ in the original space. 


\section{A.2. Proof of Proposition 2.2}

First of all, on the set $\{\omega: \Gamma(\omega)<C(\omega)\}$, by the fact that both $\Lambda$ and $B$ are hitting times, we can write $\Lambda=\Gamma+\Lambda \circ \Theta(\Gamma)$ and $B=\Gamma+B \circ \Theta(\Gamma)$, where $\Theta$ is the shift operator (see, e.g., Peskir and Shiryaev [18] page 128). Hence we can use the strong Markov property of $X$ at time $\Gamma$ to get

(A.5)

$$
\begin{aligned}
\mathbb{E}^{x}\left[1_{\{\Gamma<C\}} e^{-\rho \Lambda} f\left(X_{\Lambda}\right) 1_{\{\Lambda<B\}}\right] & =\mathbb{E}^{x}\left[1_{\{\Gamma<C\}} e^{-\rho \Gamma} \mathbb{E}^{x}\left[\left(1_{\{\Lambda \circ \Theta(\Gamma)<B \circ \Theta(\Gamma)\}} e^{-\rho(\Lambda \circ \Theta(\Gamma))} f\left(X_{\Gamma+\Lambda \circ \Theta(\Gamma)}\right)\right) \mid \mathcal{F}_{\Gamma}\right]\right] \\
& =\mathbb{E}^{x}\left[1_{\{\Gamma<C\}} e^{-\rho \Gamma} \mathbb{E}^{X_{\Gamma}}\left[1_{\{\Lambda<B\}} e^{-\rho \Lambda} f\left(X_{\Lambda}\right)\right]\right] .
\end{aligned}
$$

for any function $f$ satisfying (A.1).

With this at hand, we continue the simplification of $J_{2}(x)$ in (2.12):

$$
\begin{aligned}
J_{2}(x)-g_{1}(x) & =\mathbb{E}^{x}\left[1_{\{C<\Gamma\}} e^{-\rho C}\left(\gamma-g_{1}\left(X_{C}\right)\right)\right. \\
& \left.+1_{\{\Gamma<C\}}\left\{e^{-\rho \Gamma}\left(g_{2}\left(X_{\Gamma}\right)-g_{1}\left(X_{\Gamma}\right)\right)-e^{-\rho(\Lambda \wedge B)} g_{2}\left(X_{\Lambda \wedge B}\right)+\frac{1}{1+\eta} e^{-\rho \Lambda} V_{3}\left(X_{\Lambda}\right) 1_{\{\Lambda<B\}}\right\}\right]
\end{aligned}
$$

The last two terms in the preceding equation is

$$
\begin{aligned}
& \mathbb{E}^{x}\left[1_{\{\Gamma<C\}}\left\{e^{-\rho \Lambda}\left(-g_{2}\left(X_{\Lambda}\right)+\frac{1}{1+\eta} V_{3}\left(X_{\Lambda}\right)\right) 1_{\{\Lambda<B\}}-e^{-\rho B} g_{2}\left(X_{B}\right) 1_{\{\Lambda>B\}}\right\}\right] \\
= & \mathbb{E}^{x}\left[1_{\{\Gamma<C\}} e^{-\rho \Gamma} \mathbb{E}^{X_{\Gamma}}\left\{1_{\{\Lambda<B\}} e^{-\rho \Lambda}\left(-g_{2}\left(X_{\Lambda}\right)+\frac{1}{1+\eta} V_{3}\left(X_{\Lambda}\right)\right)-1_{\{\Lambda>B\}} e^{-\rho B} g_{2}\left(X_{B}\right)\right\}\right] \\
= & \mathbb{E}^{x}\left[1_{\{\Gamma<C\}} e^{-\rho \Gamma}\left(-g_{2}\left(\Lambda^{*}\right)+\frac{1}{1+\eta} V_{3}\left(\Lambda^{*}\right)\right) \mathbb{E}^{X_{\Gamma}}\left[1_{\{\Lambda<B\}} e^{-\rho \Lambda}\right]-g_{2}\left(B^{*}\right) \mathbb{E}^{X_{\Gamma}}\left[1_{\{\Lambda>B\}} e^{-\rho B}\right]\right],
\end{aligned}
$$

where in the first equality we use (A.5) for the appropriate functions in place of $f$ and in the second equality we note that under the assumption that $X_{\Gamma}$ is in $\left(B^{*}, \Lambda^{*}\right)$ together with path continuity of $X$, we have $X_{B}=B^{*}$ and $X_{\Lambda}=\Lambda^{*}$. Combining the pieces and using the notation (2.15) we obtain

$$
J_{2}(x)-g_{1}(x)=\mathbb{E}^{x}\left[1_{\{C<\Gamma\}} e^{-\rho C}\left(\gamma-g_{1}\left(X_{C}\right)\right)+1_{\{\Gamma<C\}} e^{-\rho \Gamma} Q\left(X_{\Gamma}\right)\right]
$$

where $Q(x)$ is defined as in (2.17). Hence to find $V_{2}(\cdot)$ is now reduced to solving the optimal stopping problem and this completes the derivation.

\section{References}

[1] L. H. R. Alvarez. On the properties of r-excessive mappings for a class of diffusions. Annals of Applied Probability, 13 (4):1517-1533, 2003.

[2] M. J. Brennan and E. S. Schwartz. Evaluating natural resource investments. Journal of Business, 58:135-157, 1986.

[3] P. D. Childs, D. C. Mauer, and S. H. Ott. Interactions of corporate financing and investment decisions: The effect of agency conflicts. Journal of Financial Economics, 76:667-690, 2005.

[4] S. Dayanik and I. Karatzas. On the optimal stopping problem for one-dimensional diffusions. Stochastic Processes and their Applications, 107 (2):173-212, 2003.

[5] E. Dynkin. Markov processes, Volume II. Springer Verlag, Berlin, 1965

[6] M. Egami. A framework for the study of expansion options, loan commitments and agency costs. Journal of Corporate Finance, 15 (3):345-357, 2009.

[7] E. Ekström. Properties of game options. Math. Meth. Oper. Res., 63:221-238, 2006.

[8] Pu Gong, Zhiwei He, and Song-Ping Zhu. Pricing convertible bonds based on a multi-stage compound-option model. Physica A: Statistical Mechanics and its Applications, 366:449-462, 2006.

[9] C. A. Hennessy. Tobin's q, debt overhang, and investment. Journal of Finance, 59:1717-1742, 2004.

[10] C. A. Hennessy and Y. Tserlukevich. Taxation, agency conflicts and the choice between callable and convertible debt. Journal of Economic Theory, 143 (1):374-404, 2008.

[11] Y. Kifer. Game options. Finance and Stochastics, 4:443-463, 2000.

[12] T. Korkeamaki and W. T. Moore. Capital investment timing and convertible debt financing. International Review of Economics and Finance, 13 (1):75-85, 2004 
[13] E. Lyandres and A. Zhdanov. Convertible debt and investment timing. Working paper, 2006.

[14] D. C. Mauer and S. Sarkar. Journal of banking and finance. Real options, agency conflicts, and optimal capital structure, 29:1405-1428, 2005.

[15] A. S. Mello and J. E. Parsons. Measuring the agency cost of debt. Journal of Finance, 47:1887-1903, 1992.

[16] B. Øksendal. Stochastic Differential Equations: An Introduction with Applications, 5th Edition. Springer, Berlin, Heidelberg, 1998.

[17] B. Øksendal and A. Sulem. Applied Stochastic Control of Jump Diffusions. Springer-Verlag, Berlin, Heidelberg, 2004.

[18] G. Peskir and A. N. Shiryaev. Optimal stopping and Free-Boundary Problems (Lectures in Mathematics, ETH Zürich). Birkhauser, Basel, 2006.

[19] S. Sarkar. Early and late calls of convertible bonds: Theory and evidence. Journal of Banking and Finance, 27 (7):1349-1374, 2003.

[20] A. Seierstad. Stochastic Control in Discrete and Continuous Time. Springer, Berlin, Heidelberg, 2008.

[21] M. Sîrbu and S. E. Shreve. A two-person game for pricing convertible bonds. SIAM J. Control Optim., 45:1508-1539, 2006.

[22] K. Yagi, R. Takashima, H. Takamori, and K. Sawaki. Timing of convertible debt financing and investment. Working paper, University of Tokyo, 2008. 